\title{
POWER EFFICIENT TASK SCHEDULING MECHANISM IN CLOUD ENVIRONMENT: A REVIEW
}

\author{
Abhilasha $^{(1)}$, Dr. Anupama Gupta ${ }^{(2)}$ \\ ${ }^{(1)}$ Research Scholar, Department of Computer Engineering, LLRIET, Moga. \\ abhilasha.1100@gmail.com \\ ${ }^{(2)}$ H.O.D, Department of Computer Science \& Engineering, LLRIET, Moga. \\ dranupamagupta@IIriet.ac.in
}

\begin{abstract}
Cloud Computing is being used widely all over the world by many IT companies as it provides various benefits to the users like cost saving and ease of use. However, with the growing demands of users for computing services, cloud providers are encouraged to deploy large datacenters which consume very high amount of energy and also contribute to the increase in carbon dioxide emission in the environment. Therefore, we require to develop techniques which will help to get more environment friendly computing i.e. Green Cloud Computing. In this paper, we have reviewed the existing mechanisms and a new strategy to reduce the carbon dioxide emissions in federated Cloud ecosystems. More specifically, we propose a solution that allows providers to determine the best green destination to reduce the carbon dioxide emissions of the whole federated environment.
\end{abstract}

\section{Keywords}

Cloud Computing, Virtual Machine, Data Center, Data Center Broker, Host.

\section{INTRODUCTION}

Cloud computing is an on demand service in which shared resources, information, software and other devices are provided according to the clients requirement at specific time. It's a term which is generally used in case of Internet. The whole Internet can be viewed as a cloud. Capital and operational costs can be cut using cloud computing. In case of Cloud computing services can be used from diverse and widespread resources, rather than remote servers or local machines. There is no standard definition of Cloud computing. Generally it consists of a bunch of distributed servers known as masters, providing demanded services and resources to different clients known as clients in a network with scalability and reliability of datacenter. The distributed computers provide on-demand services. Services may be of software resources (e.g. Software as a Service, SaaS) or physical resources (e.g. Platform as a Service, PaaS) or hardware/infrastructure (e.g. Hardware as a Service, HaaS or Infrastructure as a Service, laaS ). Amazon EC2 (Amazon Elastic Compute Cloud) is an example of cloud computing services [2].

\section{CLOUD COMPONENTS}

A Cloud system consists of 3 major components such as clients, datacenter, and distributed servers. Each element has a definite purpose and plays a specific role.

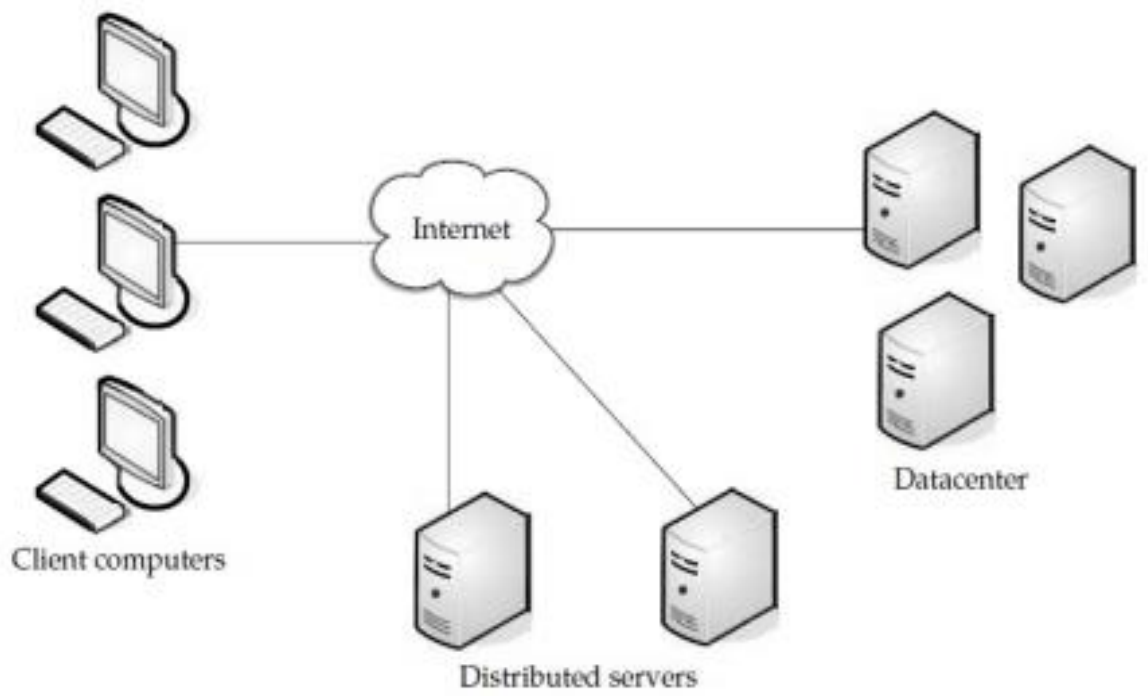

Figure 1. Cloud Components 


\section{Clients}

End users interact with the clients to manage information related to the cloud. Clients generally fall into three categories as given in [1]:

Mobile: Windows Mobile Smartphone, smartphones, like a Blackberry, or an iPhone.

Thin: They don't do any computation work. They only dispaly the information. Servers do all the works for them. Thin clients don't have any internal memory.

Thick: These use different browsers like IE or mozilla Firefox or Google Chrome to connect to the Internet cloud. Now-adays thin clients are more popular as compared to other clients because of their low price, security, low consuption of power, less noise, easily replaceble and repairable etc.

\section{Datacenter}

Datacenter is nothing but a collection of servers hosting different applications. A end user connects to the datacenter to subscribe different applications. A datacenter may exist at a large distance from the clients. Now-a-days a concept called virtualisation is used to install a software that allow multiple instances of virtual server applications.

\section{Distributed Servers}

Distributed servers are the parts of a cloud which are present throughout the Internet hosting different applications. But while using the application from the cloud, the user will feel that he is using this application from its own machine.

\section{GREEN CLOUD COMPUTING}

Cloud Computing is being used widely all over the world by many IT companies as it provides various benefits to the users like cost saving and ease of use. However, with the growing demands of users for computing services, cloud providers are encouraged to deploy large datacenters which consume very high amount of energy and also contribute to the increase in carbon dioxide emission in the environment. Therefore, we require to develop techniques which will help to get more environment friendly computing i.e. Green Cloud Computing. Therefore a new technique to reduce the carbon emission and energy consumption in the distributed cloud datacenters having different energy sources and carbon footprint rates known as Green Computing.

Green Cloud Computing is becoming important due to the increasing concerns about environmental issues by the large Internet and cloud service providers. In fact, estimations of energy consumption by information and communication technologies (ICT) point to an expressive $1.3 \%$ of the total energy consumed worldwide. Aligned with Green Cloud Computing, Green Networking is a recent field of research that aims to improve the energy efficiency of the networks via new techniques of network design and operations. Cloud infrastructures employ virtualization techniques for the sharing of resources. Such techniques allow the management of virtual machines (VMs), software artifacts able to emulate full hardware platforms. VMs share the processing resources present in their hosting servers such as central processing units (CPUs), memory, persistent storage, and net-work interfaces. The placement of VMs is the problem of selecting the servers where the VMs are allocated in such a way that some performance metrics are optimized. Examples of such metrics include energy consumed, quality of service, fault tolerance, communication latency, and load balancing.

\section{RELATED WORK}

Yuyang Peng et. al (2016) has proposed an evaluation energy efficient virtual machine allocation and genetic based algorithm based meta heuristic which support a power aware VM request allocation of multiple sustainable cloud data centers. This approach provides a novel metrics which diagnoses the efficiency of power of each cloud datacenters.

Huigui Rong et. al (2016) has reviewed the progress of energy-saving technologies in high-performance computing, energy conservation technologies for computer room and renewable energy applications during the construction and operation of data centers. From multiple perspectives of energy consumption and environment protection, a comprehensive set of strategies are proposed to maximize data centers efficiency and minimize the environment an impact. This research also provides energy-saving trends for data centers in the future.

Fahimeh Farahnakian et. al (2015) has investigated the effectiveness of VM and host resource utilization predictions in the VM consolidation task using real workload traces. The experimental results show that the approach provides substantial improvement over other heuristic algorithms in reducing energy consumption, number of VM migrations and number of SLA violations.

Maurizio Giacobbe et. al (2015) has applied a new strategy to reduce the carbon dioxide emissions in federated Cloud ecosystems. More specifically, they have discussed a solution that allows providers to determine the best green destination where virtual machines should be migrated in order to reduce the carbon dioxide emissions of the whole federated environment.

Moona Yakhchi et. al (2015) has presented an approach based on Cuckoo Optimization Algorithm (COA) to detect overutilized hosts. They have employed The Minimum Migration Time (MMT) policy to migrate Virtual Machines (VMs) from

7254 | P a g e

A u gust, 2016
Council for Innovative Research

w w w. cir world. co m 
the over-utilized hosts to the under-utilized hosts. The results generated by Cloud sim simulator, demonstrated that the proposed approach has lowest energy consumption compared to the other famous algorithms.

Chenxi Qiu et. al (2015) has stated the functioning of CSB (Cloud Service Brokerage) as an intermediary between tenants and cloud providers that can bring about great benefits to the cloud market. CSBs buy the cloud resources, i.e., servers, with lower prices from cloud providers and sell the resources to the tenants with higher prices. To maximize its own profit, a CSB may distribute tenants' requests to the clouds that waste energy resources.

Yibin Li et. al (2015) has proposed a novel Energy-aware Dynamic Task Scheduling (EDTS) algorithm based on DVS (Dynamic Voltage Scaling) to minimize the total energy consumption for smart phones, while satisfying stringent time constraints and the probability constraint for applications.

YunNi Xia et. al (2015) has presented a novel stochastic framework for energy efficiency and performance analysis of DVS-enabled cloud. This framework uses virtual machine request arrival rate, failure rate, repair rate, and service rate of datacenter servers as model inputs.

Mahesh b. Nagpure et. al (2015) has proposed dynamic resource allocation system which allocated resources to cloud user. The skewness algorithm measure uneven utilization of multiple resources of each VMs and accordingly balances across VMs. By minimizing skew value of each VM, we can easily combine different multiple resources and improve resource utilization of server.

S. Yakhchi et. al (2015) has proposed a novel power aware load balancing method, named ICAMMT to manage power consumption in cloud computing data centers. They have exploited the Imperialism Competitive Algorithm (ICA) for detecting over utilized hosts and then we migrate one or several virtual machines of these hosts to the other hosts to decrease their utilization.

Yu-Wen Chen et. al (2015) has proposed a cloud based framework to provide a customer-oriented energy management as a service (EMaaS) for green communities, which are formed as virtual retail electricity providers (REPs) by involved DERs (Distributed Energy Resources) providers. It can be adopted by existing REPs or utilities. For each green community, the multiperiod global cost is minimized to promote renewable energy, and renewable energy consumption is stabilized to enhance integration.

Anna Agustí Torra et. al (2015) has debated about the incentives that customers and data centers can adopt such measures and propose a new service type and pricing scheme that is economically attractive and technically realizable. Simulation results based on real measurements confirm that the scheme can achieve additional energy savings while preserving service performance and the interests of data centers and customers.

Doshi Chintan Ketankumar et. al (2015) has proposed a green cloud broker for resource procurement problem by considering the metrics of energy efficiency and environmental friendly operations of the cloud service provider. They have used mechanism design methods to decide the allocation and payment for the submitted job dynamically.

Shaoming Chen et. al (2015) has quantitatively analyzed the impact of server consolidation and temperature of cooling water on the total electricity and server maintenance costs in hybrid cooling data centers. To minimize the total costs, we proposed to maintain sweet temperature and available sleeping time threshold (ASTT) by which a joint cost optimization can be satisfied.

Niangjun Chen et. al (2015) has focused on "greening" demand response in multi-tenant data centers by designing a pricing mechanism through which the data center operator can efficiently extract load reductions from tenants during emergency periods for EDR(Emergency Demand Response).

S.R. Sivarasu et. al (2015) has described that the regional renewable resources can effectively beutilized for powering the MG (Micro Grid) to supply fixed and deferrable loads.Communication between residential consumers during energy transfer through MG and communication between MG to utility gridis also emphasized. A case study is presented to show the demand and load pattern so selective residential consumers.

Bharti Wadhwa et. al (2014) has proposed a new technique to reduce the carbon emission and energy consumption in the distributed cloud datacenters having different energy sources and carbon footprint rates. They have used the carbon footprint rate of the datacenters in distributed cloud architecture and the concept of virtual machine allocation and migration for reducing the carbon emission and energy consumption in the federated cloud system.

Sonika P Reddy et. al (2014) has presented a system that handles real-time and non-real-time tasks in an energy efficient method without compromising much on neither reliability nor performance. Of the three processors, two processors i.e. the first and second, handle real-time tasks, using earliest-Deadline-First (EDF) and Earliest-Deadline-Late (EDL) scheduling algorithms respectively. On the third processor, the non-real-time tasks are scheduled using the First Come First Served (FCFS) scheduling algorithm and the tasks are run at an energy efficient frequency.

7255 | P a g e

A u gust , 2016
Council for Innovative Research

w w w. cirworld. com 
Lucio A. Rocha et. al (2014) has stated a hybrid optimization model that allows a cloud service provider to establish virtual machine placement strategies for its data centers in such a way that energy efficiency and network quality of service are jointly optimized. The proposed strategy allows cloud providers to reach a balance between the energy efficiency of their infrastructures and the network quality of service they offer to their customers.

Samiran Roy et. al (2014) has proposed a green cloud enabled framework which envisioned the energy efficient way of a minimal discharge and rectification of the problem of high carbon production so as to increase the profit margin.

Yi-Ju Chiang et. al (2013) has discussed an efficient green control (EGC) algorithm for solving constrained optimization problems and making costs/performances tradeoffs in systems with different power-saving policies. Simulation results show that the benefits of reducing operational costs and improving response times can be verified by applying the powersaving policies combined with the proposed algorithm as compared to a typical system with a same performance guarantee.

\section{PROBLEM FORMULATION}

Green Computing refers to the attempts to maximize the use of power consumption and energy efficiency and to minimize the cost and CO2 emission. Development of new computing models, computer systems, and applications having low cost and low energy consumption are the primary purposes of Green Computing. As we know the existing system has three processors. The first and second processors will handle real time tasks and the third processor will handle only non-real time tasks. If we consider that a task real time task $\mathrm{T} 1$ arrives at P1 while it is idle then this task will execute on P1 itself and that too at maximum frequency. Maximum frequency ensures that the task will run to its completion and therefore for such a task we need not keep a backup. Hence If Task T1 executes successfully on P1 then back up of task T1 at P2 is no longer required and therefore, $\mathrm{P} 2$ is using the power unnecessarily.

Another issue which needs to be addressed is that the waiting time for next Tasks T2, T3, and T4...so on will keep on increasing as they will enter the queue. Although we have the processor P2 which is sitting idle. It is so because as per the research paper author $\mathrm{P} 2$, the second processor no doubt will handle real time processes but it will activate only when any task fails on $\mathrm{P} 1$. So in other words the second processor acts only as a backup device but it actually will never share the load of the first processor. This also implies that though the system has two processors to handle real time tasks but in actual practice only one task will get executed at one time.

Analysis of the system further reveals another flaw that what if no non-real time task arrives or only non-real tasks arrive, still then the power will be consumed by the three processors. It will be so because each processor is built to handle specific task.

\section{OBJECTIVES}

1. To study and implement the performance of existing green computing algorithms in cloud environment.

2. To design the optimized green computing model in cloud environment.

3. To develop the proposed algorithm for improving the overall power consumed by the datacenter in CloudSim Framework.

4. To compare the performance of the algorithm with the proposed algorithm in terms of power consumed, cost incurred and utilization of resources.

\section{REFERENCES}

[1] S. M. G. Y. F. P. Moona Yakhchi, "Proposing a Load Balancing Method Based on Cuckoo Optimization Algorithm for Energy Management in Cloud Computing Infrastructures," Borujerd, Iran, 2015.

[2] Y.-J. Chiang, "An Efficient Green Control Algorithm in Cloud Computing for Cost Optimization," in IEEE.

[3] T. P. P. L. J. P. H. T. Fahimeh Farahnakian, "Utilization Prediction Aware VM Consolidation Approach for Green Cloud Computing," Turku, Finland, 2015.

[4] A. V. Bharti Wadhwa, "Carbon Efficient VM Placement and Migration Technique for Green Federated Cloud Datacenters," Chandigarh,India, 2014.

[5] S. G. Samiran Roy, "The Green Cloud Effective Framework: An Environment Friendly Approach Reducing CO2 Level," Kalyani, India, 2014.

[6] C. H. K. S. Sonika P Reddy, "Energy Aware Scheduling of Real-Time and Non Real-Time Tasks on Cloud Processors," Bangalore, India, 2014.

$7256 \mid$ P a g e

A u gust , 2016
Council for Innovative Research w w w. cirworld. com 
[7] M. Z. L. S. P. a. Q. Z. YunNi Xia, "A Stochastic Approach to Analysis ofEnergy-Aware DVS-Enabled Cloud Datacenters," 2015.

[8] P. D. M. Mahesh B. Nagpure, "An Efficient Dynamic Resource Allocation Strategy for VM Environment in Cloud," Nagpur, India, 2015.

[9] Y. J. Chiang, Y. C. Ouyang and C.-. H. Hsu, "An Efficient Green Control Algorithm in Cloud Computing for Cost Optimization," 2014.

[10] B. Wadhwa and A. Verma, "Carbon Efficient VM Placement and Migration Technique for Green Federated Cloud Datacenters," Chandigarh,India, 2014.

[11] S. P. Reddy and C. H K S, "Energy Aware Scheduling of Real-Time and Non Real-Time Tasks on Cloud Processors," Bangalore, India, 2014.

[12] S. Roy and S. Gupta, "The Green Cloud Effective Framework: An Environment Friendly Approach Reducing CO2 Level," Kalyani, India, 2014.

[13] Y. Xia, M. Zhou, X. Luo, S. Pang and Q. Zhu, "A Stochastic Approach to Analysis ofEnergy-Aware DVS-Enabled Cloud Datacenters," 2015.

[14] B. M. Nagpure, P. Dahiwale and P. Marbate, "An Efficient Dynamic Resource Allocation Strategy for VM Environment in Cloud," Nagpur, India, 2015.

[15] M. Yakhchi, S. M. Ghafari, S. Yakhchi, M. Fazeli and A. Patooghi, "Proposing a Load Balancing Method Based on Cuckoo Optimization Algorithm for Energy Management in Cloud Computing Infrastructures," Borujerd, Iran, 2015.

[16] F. Farahnakian, T. Pahikkala, P. Liljeberg, J. Plosila and H. Tenhunen, "Utilization Prediction Aware VM Consolidation Approach for Green Cloud Computing," Turku, Finland, 2015. 